retardation in the rate of growth of the hepatic cell cytoplasm.

Department of Anatomy,

University, Glasgow, W.2. Sept. 28.

'Chalkley, H. W., J. Nat. Cancer Inst., 4, 47 (1943).

${ }^{2}$ n'Connor, R. J., J. Embryol. Exp. Morph., 1, 105 (1953).

\section{Synthesis of Long-Chain Fats by Bacteria isolated from Human Fæces}

WE have recently isolated an organism from the stool of an adult human subject that is capable of synthesizing significant quantities of fatty material, which becomo visible on the surface, from a fat-free medium. Tho fat is neutral in character, liberating fatty acids on hydrolysis, and can be removed from the surface by dissolving it in ether. It is colourless and melts below body temperature. Some of its properties are shown in Table 1. Relatively little fat is present in the medium and organisms, and has not so far been investigated.

Table 1

Saponiftcation value $190 \quad$ Reichert value $\quad 42 \cdot 1$

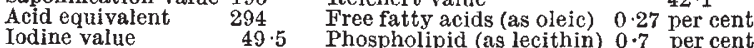

The fat was prepared by inoculating a fat-free glucose peptone medium and allowing the fat to accumulate on the surface. The organisms were Gram-positive, and were observed in short chains and pairs. The organisms do not retain sudan black, but the fat produced in the medium stains readily with 50 per cent sudan black or sudan IV. (The organisms appear to resemble Streptococcus taecalis.) The growth of bacteria and the synthesis of fat are stimulated by the presence of folic acid as shown in Fig. 1.

These organisms were obtained from a patient with steatorrhoea under treatment with folic acid, and from the amounts obtained under these in vitro conditions, namely, $200 \mathrm{mgm}$. in four days in $10 \mathrm{ml}$. of medium, it would appear that fat synthesis of this

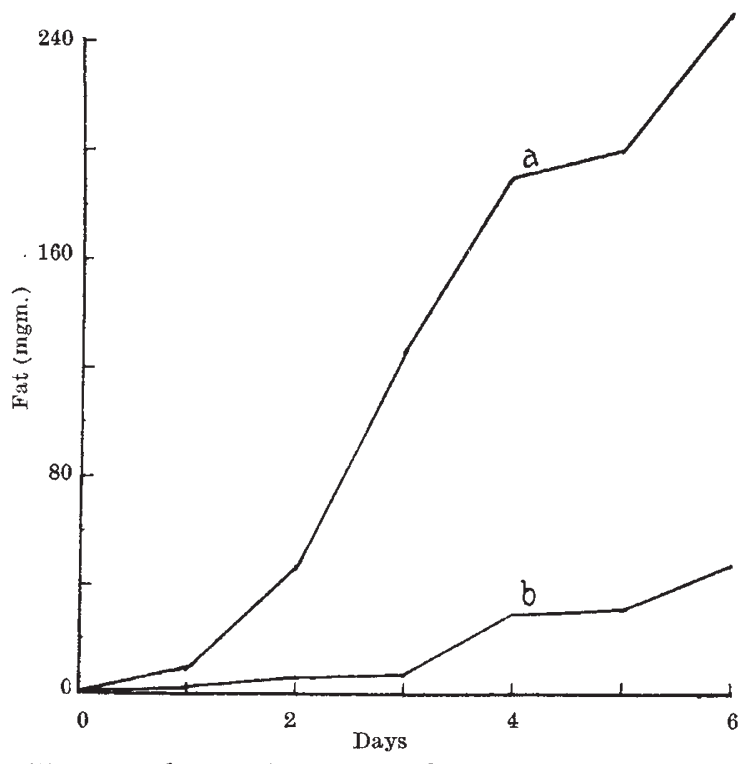

Fig. 1. $10 \mathrm{ml}$. glucose/peptone in each tube. (a) With folic acid ; (b) without folic acid type might make a significant contribution to fæcal fat. This possibility is being studied in other patients, and at the same time the properties of the fat and the factors affecting the quantity of fat formed are being more fully investigated.

H. G. SaMmons

D. J. VAUGhan

A. C. Frazer

Department of Pharmacology,

University of Birmingham, and

Metabolic Unit

Little Bromwich General Hospital, Birmingham 9.

Aug. 23.

\section{Amino-Acid Patterns of Urine and Blood Plasma in a Cystinuric Labrador Dog}

IT was shown by Lassaigne more than 130 years ago that cystine calculi may occur in the urinary tract of dogs. Canine cystinuria has since been recognized widely. Thus, more recently, 3 per cent of urinary calculi removed surgically from dogs in Holland and in Denmark ${ }^{1}$ were composed of cystine, and 18 per cent in England2, where White found them to be the commonest type (55 per cent of cases) causing urethral impaction. Canine cystinuria, with or without calculus formation, is sajd to be common also in France. Cystine calculi have been recorded from many breeds of $\mathrm{dogs}^{2-4}$, but not hitherto from the Labrador. Furthermore, no study of the aminoacid patterns of the urine and plasma has previously been described; and hence it was not known if canine cystinuria is etiologically analogous with human cystinuria. If it were so, the usefulness of the cystinuric dog as a model for investigation of the human condition would be enhanced

The subject of this communication is a male Labrador which first developed acute urinary retention in June 1951 when seven years old. Numerous small vesical calculi, and some impacted in the urethra near the penile bone, were removed surgically. A similar attack followed in November 1952, and also required urgent surgery. The animal was then placed on a reduced protein diet, and daily potassium citrate to alkalinize the urine; but, in spite of this, another crisis requiring surgical intervention occurred in October 1954.

Dent and Rose ${ }^{5}$, in a study of fifteen cases of human cystinuria by means of two-dimensional paper chromatography, found that excessive urinary excretion of lysine invariably accompanied cystine, and that abnormal excretions of arginine and ornithine varied. The opportunity was therefore taken, after the second attack in this dog, of determining whether or not the amino-acid patterns in the urine and the plasma differed from those in human cystinuria.

One specimen of urine was obtained from the dog in November 1954, and a second specimen five weeks later. A sample of plasma was obtained with the second specimen of urine. Both specimens of urine gave strongly positive reactions for cystine with the sodium cyanide/nitroprusside test, and the typical cystine crystals were observed in the deposits after centrifuging.

The plasma was deproteinized, and the resultant liquid and the two specimens of urine desalted, oxidized and chromatographed by methods outlined 